\title{
Brevundimonas Vesicularis: Unusual Cause of Bacteraemia in Otherwise Healthy 8 Months Old Premature Child: Case Report
}

By Anood Al Rawahi \& Hilal Al Hashami

Abstract- Brevundimonas vesicularis, a non-fermenting gram-negative bacterium, has rarely been diagnosed as a cause of infection in an otherwise healthy child. In this report, we describe Brevundimonas vesicularis bacteremia, in an 8 months old healthy girl who was treated successfully with intravenous piperacillin-tazobactam.

Strictly as per the compliance and regulations of:

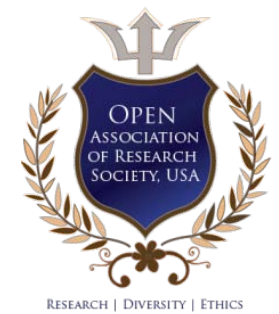

(C) 2020. Anood Al Rawahi \& Hilal Al Hashami. This is a research/review paper, distributed under the terms of the Creative Commons Attribution-Noncommercial 3.0 Unported License http://creativecommons.org/licenses/by-nc/3.0/), permitting all noncommercial use, distribution, and reproduction in any medium, provided the original work is properly cited. 


\title{
Brevundimonas Vesicularis: Unusual Cause of Bacteraemia in Otherwise Healthy 8 Months Old Premature Child: Case Report
}

\author{
Anood Al Rawahi ${ }^{\alpha} \&$ Hilal Al Hashami ${ }^{\sigma}$
}

Abstract- Brevundimonas vesicularis, a non-fermenting gramnegative bacterium, has rarely been diagnosed as a cause of infection in an otherwise healthy child. In this report, we describe Brevundimonas vesicularis bacteremia, in an 8 months old healthy girl who was treated successfully with intravenous piperacillin-tazobactam.

\section{INTRODUCTION}

G ram-negative bacteremia (GNB) is a common clinical encounter in children with a history of recurrent hospital admission

or

immunocompromised children. Growth of GNB from a sterile site should be considered carefully even in an otherwise healthy children with no predisposing risk factors for such infection. We present a case of Brevundimonas vesicularis bacteremia in otherwise healthy eight months old premature baby girl with a history of prolonged NICU admission without any prematurity complications.

\section{il. Case Presentation}

Eight months old twin one of monochorionic diamniotic twins with corrected age of 6 months, born at 31 weeks of gestation via elective caesarian section required neonatal intensive care unit ( $\mathrm{NICU})$ admission for 20 days due to low birth weight. She remained asymptomatic after discharge from NICU. She brought by her parents to the emergency department with a history of cough, fever and increase work of breathing for a one-week duration. Cough is wet, with no posttussive vomiting. No cyanosis or episodes of apnea. On examination, she was sick looking, febrile with temperature $38.6 \mathrm{C}$, respiratory rate 52 breath per minute, heart rate 150 beats per minute. The chest examination revealed a wheezy chest. Her investigations showed: total white blood cells WBC: $15.3 \times 10^{9}$ cells/L neutrophils $12.1 \times 10^{9} / \mathrm{L}$, lymphocytes $2.6 \times 10^{9} / \mathrm{L}$, monocytes $0.4 \times 10^{9} / \mathrm{L}$, eosinophils $0.1 \times 10^{9} / \mathrm{L}$, basophils $0.1 \times 10^{9} / \mathrm{L}$. blood gas: $\mathrm{pH}=7.41, \mathrm{pCO} 2=$ $38 \mathrm{mmHg}, \mathrm{pO} 2=89.9 \mathrm{mmHg}$, bicarbonate $=24 \mathrm{mmol} / \mathrm{L}$. The respiratory viral panel came positive for the respiratory syncytial virus (RSV). Peripheral blood culture collected along with catheterized urine culture. Chest Xray showed: right-sided infiltrate (figure1). The initial clinical impression was chest infection based on tachypnea, chest $\mathrm{x}$-ray findings, and high total white blood cells. She was admitted for intravenous antibiotics.

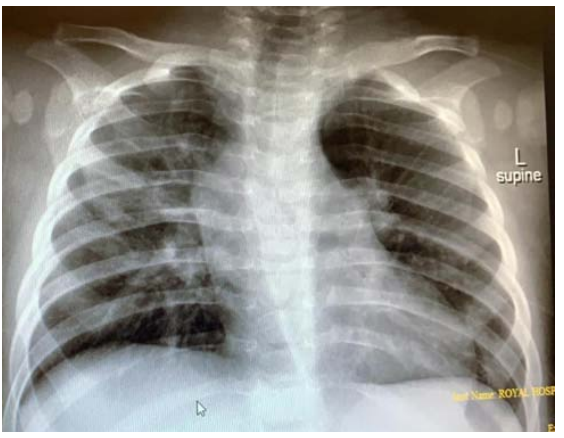

Figure 1: Chest $x$-ray of the child

She was started empirically on intravenous Augmentin $30 \mathrm{mg}$ three times a day. She required frequent suctioning on the first day of admission along with nasogastric tube (NGT) feeding of $75 \mathrm{ml}$ every 2 hours along with PRN salbutamol nebulization as she

Author a: Pediatric Intern, Royal Hospital, Muscat, Oman.

e-mail: anoodsalrawahi@gmail.com

Author o: Pediatrics Infectious Diseases consultant, Royal Hospital, Muscat, Oman. continued to have a wheezy chest. By the second day of admission, she continued to spike a fever and had reduced activities despite improvement in her respiratory status.

On the $3^{\text {rd }}$ day of admission, the blood culture flagged positive for gram-negative bacilli sensitive to piperacillin + tazobactam, gentamycin, and cefepime, resistant to ciprofloxacin and ceftazidime. Based on the sensitivity report; augmentin changed to intravenous 
piperacillin-tazobactam 0.95 grams 8 hourly. Two days later, the final identification of the gram-negative bacilli reported as Brevundimonas Vesicularis. Clinically, the patient improved after the $2^{\text {nd }}$ day of intravenous piperacillin-tazobactam. She became a febrile, her activities improved, and her general condition also improved. She did not show any signs of meningitis, urine analysis and culture were negative. Repeated blood culture 48 hours after starting her on piperacillintazobactam came negative for bacterial growth. As this organism is more common in immunocompromised patients, the child was investigated for immunodeficiency. The following investigations were done: immunoglobulin $\operatorname{lgG} 7.9 \mathrm{~g} / \mathrm{L}(2.02$ - 9.5), immunoglobulin $\mathrm{lgA} 0.32 \mathrm{~g} / \mathrm{L}(.08-.91)$, immunoglobulin $\lg \mathrm{M} 0.83 \mathrm{~g} / \mathrm{L}$ (.17-1.5). Lymphocytes subset analysis panel including; Total lymphocytes $5.73 \times 10^{9}$, T-cell $(\mathrm{CD} 3+)=5.01 \times 10^{9}$, B-cell $(\mathrm{CD} 19+)=1.26 \times 10^{9}, \mathrm{~T}$ helper $(\mathrm{CD} 3+/ \mathrm{CD} 4+)=3.94 \times 10^{9}, \quad$ T-cytotoxic $(\mathrm{CD} 3+/ \mathrm{CD} 8+)=1.01 \times 10^{9}, \mathrm{CD} 4: \mathrm{CD} 8$ ratio $=3.90$ $\times 10^{9}$, NK-cells $(\mathrm{CD} 3-/ \mathrm{CD} 56+)=0.26 \times 10^{9}$. All her immunological investigations were within normal and were not suggestive of primary immunodeficiency.

She discharged after completing ten days of intravenous antibiotics. On subsequent out-patient follow-up, she remains asymptomatic and is gaining weight and gaining her milestones according to her chronological age.

\section{Discussion}

The Brevundimonas are a genus of proteobacteria, gram-negative, non-fermenting, aerobic bacilli. Oxidase and catalase-positive. Two species of Brevundimonas classified under the genus pseudomonas then it has been re-classified by Seger et al. as Brevundimonas vesicularis and Brevundimonas diminuta1. There have been many cases reports of Brevundimonas infection isolated from different sites such as skin and soft tissues, urinary tract infections, liver abscess, meningitis, and peritonitis. The predisposing factors of Brevundimonas infection are not well known. Most of the infection occurs in immunocompromised patients such as those with prolonging steroid therapy, systemic lupus erythematosus (SLE), end-stage renal disease, and malignancy. ${ }^{2}$ This organism has also been isolated from environmental samples such as soil, tap water, and hospital instruments. ${ }^{3}$

Previous case reports of the same organism showed a variety of drug susceptibility. Although it was not used frequently to treat this organism, most of the cases reported showed no resistance to cotrimaxazole, which also observed in our case. Piperacillin+ tazobactam is the most common medication which used in most of the reported cases. Shang et al. observed that the organism was sensitive to aminopenicillins, penicillins, cephalosporins, carbapenems, and it was resistant to aztreonam, ceftazidime, and ciprofloxacin².

Our reported case was sensitive to piperacillin + tazobactam, gentamycin, and cefepime. It was resistant to ciprofloxacin and ceftazidime. The child improved with piperacillin + tazobactam treatment course for a total of 10 days' duration with no complications. Karadag et al, reported in his study of a 29-week-old neonate who developed early neonatal sepsis due to the same organism complicated by persistent meningitis and lymphadenopathy. ${ }^{4}$

The infection caused by Brevundimonas can have different presentations. One reported case of Brevundimonas septicemia complicated by bilateral pneumothorax and empyema in an eight-months-old infant who presented with fever, rapid breathing, and poor oral intake required chest tube insertion, treated successfully with Cefoperazone and levofloxacin then discharged after two weeks of treatment. ${ }^{5}$ Another case reported in an immunocompetent young male, presented with liver abscess required drainage in addition to antimicrobial therapy of ceftriaxone followed by ampicillin/sulbactam. ${ }^{6}$ A rare presentation of septic arthritis of shoulder joint in a previously healthy toddler witch managed successfully with cefuroxime antibiotic. ${ }^{7}$

The present case report demonstrates the importance of diagnosing Brevundimonas bacteremia, particularly in otherwise a healthy child with no predisposing risk factors if the whole clinical picture cannot be explained by the viral infection.

\section{In Conclusion}

Brevundimonas causes serious infection rather than just be considered as a contamination in high-risk setting. Once it is isolated from a sterile site, it should be taken seriously and appropriate antibiotic therapy should be started. Early treatment with follow up culture is the kay to prevent morbidity and mortality related to this infection.

\section{References Références Referencias}

1. Palleroni NJ. Genus I pseudomonaceae. In: Krieg NR, Holt JG, eds. Bergey's Manual of Systematic Bacteriology. Baltimore/London: Williams and Wilkins, 1984; 141-99.

2. Shang ST, Chiu SK, Chan MC, Wang NC, Yang YS, Lin JC, et al. Invasive Brevundimonas vesicularis bacteremia: Two case reports and review of the literature. J Microbiollmmunol Infect 2012;45:468-72

3. Mondello P, Ferrari L, Carnevale G. Nosocomial Brevundimonas vesicularis meningitis. Infez Med 2006; 14:235-7.

4. Karadag N. Karagol B.S. Dursun A. Okumus N. Tanir G. Zenciroglu A. A premature neonate with early-onset neonatal sepsis owing to Brevundimonas vesicularis complicated by 
persistent meningitis and lymphadenopathy. Infection. 2012; 40: 509-515

5. Singh S, Bhatia BD. Brevundimonas Septicemia: A Rare Infection with Rare Presentation. Indian Pediatr. 2015;52(10):901. doi:10.1007/s13312-015-0743-6

6. Yoo SH, Kim MJ, Roh $\mathrm{KH}$, et al. Liver abscess caused by Brevundimonas vesicularis in an immunocompetent patient. J Med Microbiol. 2012; 61(Pt 10):1476-1479. doi:10.1099/jmm.0.045120-0

7. Sofer Y, Zmira S, Amir J. Brevundimonas vesicularis septic arthritis in an immunocompetent child. Eur $J$ Pediatr. 2007; 166(1):77-78. doi:10.1007/s00431006-0216-y 